\title{
Propuesta de mejora mediante la aplicación de la metodología de Mantenimiento Productivo Total a los servicios de mantenimiento de los Molinos SAG
}

\section{Proposal for improvement through the application of the Total Productive Maintenance methodology to the maintenance services of the SAG Mills}

\author{
Jorge L. Alfaro Rosas, Magister ${ }^{1}$, Elizabeth K. Bravo Huivin, Magister ${ }^{1}$, Richard E. Gutierrez Valera, Ingeniero ${ }^{1}$, \\ Danny S. Zelada Mosquera, Magister ${ }^{1}$, Carlos E. Mendoza Ocaña, Magister ${ }^{1}$, Jercy. Cevallos Castillo, and Juan M. \\ Deza Castillo, Magister ${ }^{1}$ \\ ${ }^{1}$ Universidad Privada del Norte, Perú,jorge.alfaro@upn.edu.pe,kristina.bravo@upn.pe,danny.zelada@upn.edu.pe, \\ carlos.mendoza@upn.edu.pe,jercy.cevallos@upn.edu.pe,juan.deza@upn.edu.pe
}

\begin{abstract}
Resumen- La presente propuesta de mejora tiene como objetivo determinar el efecto que la aplicación de la metodología de mantenimiento productivo total tiene en el servicio de mantenimiento de molinos $S A G$. Para ello, se siguió un enfoque de investigación no experimental porque no se manipulará la variable de estudio y propositiva, porque permitirá realizar una propuesta a los servicios de mantenimiento de molinos SAG basado en el TPM. En esta investigación se emplearon técnicas de recolección de información como observación, encuesta y entrevista. A continuación, se muestran los aspectos generales sobre el problema de la investigación, se describen los planteamientos teóricos relacionados con la presente propuesta de mejora, se realiza el diagnóstico situacional de la empresa, donde hace mención a los problemas existentes en el servicio de mantenimiento y sus respectivas causas raíz, esto mediante un diagrama Ishikawa se realiza el desarrollo de las herramientas y metodologías a utilizar para la solución de estas causas raíces. Por último y con toda la información analizada y recolectada; a partir del diagnóstico realizado, se presenta un análisis de resultados y discusión para poder corroborar con datos las evidencias presentadas y la mejora lograda con la propuesta en el servicio de mantenimiento de molinos SAG. Se concluye que la aplicación de la metodología de mantenimiento productivo total tiene en el servicio de mantenimiento de molinos $S A G$ tiene un efecto positivo en el servicio de mantenimiento de molinos $S A G$ dado que genera un ahorro de $S / 350$ 000,00 anuales en el servicio.
\end{abstract}

Palabras Clave-Procesos; TPM, 5S, Molinos SAG, Mantenimiento

Abstract- The present improvement proposal aims to determine the effect that the application of the total productive maintenance methodology has on the SAG mill maintenance service. For this, a non-experimental research approach was followed because the study and proposition variable will not be manipulated, because it will allow a proposal to be made to the maintenance services of $S A G$ mills based on the TPM. In this research, information gathering techniques such as observation, survey and interview were used. Next, the general aspects of the research problem are shown, the theoretical approaches related to this improvement proposal are described, the situational diagnosis of the company is carried out, where it makes mention of the existing problems in the maintenance service and their respective root causes, this through an Ishikawa diagram, the development of the tools and methodologies to be used for the solution of these root causes is carried out.
Finally and with all the information analyzed and collected; Based on the diagnosis made, an analysis of the results and discussion are presented in order to corroborate with data the evidence presented and the improvement achieved with the proposal in the SAG mill maintenance service. It is concluded that the application of the total productive maintenance methodology has in the SAG mill maintenance service has a positive effect on the $S A G$ mill maintenance service since it generates a saving of $S$ / $350,000.00$ per year in the service.

Keywords-- Processes; TPM, 5S, SAG Mills, Maintenance

\section{INTRODUCIÓN}

Durante la primera mitad del siglo XX, la solución de trituración consistía en usar una planta de chancado fino para reducir la producción mineral bruta de la mina de 300 $\mathrm{mm}$ a aproximadamente $15 \mathrm{~mm}$, un tamaño apropiado para alimentar los molinos trituradores de barras. Normalmente, los molinos trituradores de barras reducen el mineral de $15 \mathrm{~mm}$ a aproximadamente $1 \mathrm{~mm}$. Luego, se utilizaban molinos de bolas, que reducían la alimentación de $1 \mathrm{~mm}$ proveniente de los molinos trituradores de barras al tamaño necesario para la separación de minerales, de 75 a 150 micrones. Las plantas de chancado secundario tienen una disponibilidad relativamente baja (alrededor del 65\%), su mantenimiento es costoso y presentan diversos riesgos a la seguridad y salud ocupacional, especialmente en cuanto a las actividades de mantenimiento y al polvo en el aire.

A finales del siglo $\mathrm{XX}$, el chancado secundario, los molinos trituradores de barras y los molinos de bolas fueron reemplazados por molinos autógenos (AG) y semi autógenos (SAG) de gran capacidad, acompañados normalmente por molinos de bolas. Según el diccionario Webster, autógeno se define como "producido independientemente de ayuda o influencia externas". En esta aplicación de molinos, autógeno se refiere a que el tamaño de los minerales se reduce por la

Digital Object Identifier (DOI):

http://dx.doi.org/10.18687/LEIRD2021.1.1.30

ISSN: 2414-6390 ISBN: 978-958-52071-9-6 
mera acción de moverse dentro del molino, como en el caso de un molino autógeno (AG). El tamaño del mineral en movimiento dentro de un molino semiautógeno (SAG), se reduce parcialmente debido a este movimiento y mediante una agitación adicional producida por la adición de bolas de acero. Las empresas mineras para procesar el mineral que explotan usa como parte de la planta procesadora a los molinos SAG, estos molinos trituran el mineral proveniente de chancado.

Los molinos SAG tienen la forma de un cilindro gigante el cual se encuentra instalado de forma horizontal y gira a unas determinadas rpm, en su interior cuenta con bolas de acero que junto con el giro del molino reducen el diámetro del material que ingresa en el molino, también cuentan con componentes internos llamados liners los cuales presentan un índice de desgaste elevado debido a la abrasión que se genera en su interior, ya que estos liners funcionan como protectores de la parte interna del shell del molino SAG. Todos los molinos SAG poseen mantos estructurales cilíndricos que están protegidos contra la abrasión por lainers o revestimientos extraíbles y reemplazables. Este desgaste causa que las empresas mineras realicen un mantenimiento preventivo programado anualmente, para evitar que el shell sea fracturado a causa de los impactos de la carga interna de las bolas de acero y el mineral.

Por este motivo se realiza los servicios de "cambio de liners o revestimientos", en los cuales los clientes, es decir, las empresas mineras, necesitan de empresas altamente especializadas y calificadas para cumplir con la calidad de servicio requerido y los tiempos establecidos para dicho mantenimiento, ya que extender los tiempos de mantenimiento significaría pérdida de producción de la planta, cuando la planta que procesa el mineral se detiene para las tareas necesarias de mantenimiento, se interrumpe el proceso de producción y por ende el flujo de dinero de toda la mina. la vida útil y el reemplazo de los revestimientos definen el tiempo disponible para el procesamiento de minerales.

Por cada hora que el concentrador está apagado debido al cambio de revestimientos de los molinos, los activos de la mina pierden entre 50000 y 500000 dólares de ingresos, dependiendo de la ley de cabeza, el rendimiento y los precios de los productos de la mina. A la fecha existe esta necesidad de reducir el tiempo sin producir de los Molinos SAG por el alto costo que causa en toda la empresa minera debido a los cambios de revestimientos, todo este asunto ha llevado a mantener un constante estudio no tan solo en la Maquinaria y Herramientas utilizadas en él, sino también en adoptar una estrategia operacional que optimice los recursos, estrategias de mantenimiento y una óptima elección en la decisión de los equipos a utilizar para ejecutar el mantenimiento. El mercado se estima en promedio \$ 5 millones anuales al 2019 y se proyecta al 2021 en $\$ 8$ millones. El crecimiento en este sector ser aprovechado para ganar mayor participación de mercado. Para esto, se impone un cambio de estrategia del servicio, ya que uno de los principales servicios que brinda es el cambio de liners de molinos SAG.

Por ello, un nuevo "tipo de servicio" ayudaría a una mayor participación de mercado y crecimiento de la compañía. Lo mencionado en líneas anteriores, se enfoca en los siguiente: seguridad, medio ambiente, tiempos de ejecución, mejoras enfocadas y plan estratégico del mantenimiento a ejecutar que nos diferenciarán de la competencia actual en el mercado y con esta propuesta de mejora se espera que la compañía estudiada sea mejor opción para sus clientes, quienes podrán verse beneficiados por el desarrollo del mantenimiento de molinos SAG de manera que el cumplimiento de sus objetivos de gestión interna sea optimo, hay necesidades de autofinanciamiento que se considera que sean del $100 \%$.

Asimismo, dicho cumplimiento se ve enmarcado en la inversión de los colaboradores, que se detallarán a lo largo de los capítulos de esta tesis, que demostrará que se puede obtener al final de esta propuesta una cartera de clientes consolidada, contratos renovados y clientes adjudicados. El objetivo principal del mantenimiento es mantener los equipos y activos de una empresa en las mejores condiciones de funcionamiento, por ende, esto se reflejará en un buen nivel de calidad, confiabilidad y disponibilidad al menor costo posible. Estos elevados niveles se plasman directamente a la capacidad de producción y productividad y así a los beneficios económicos de la empresa. [1]

\section{A. Mantenimiento productivo total}

El mantenimiento tradicionalmente es considerado como un centro de costo, pero no es valorado por el impacto significativo que tiene en la empresa a través de la disponibilidad del equipo [2]. El Mantenimiento Productivo Total tiene un impacto considerable en puntos estratégicos de las empresas, tales como la rentabilidad [3], las inversiones de capital [4], así como el rendimiento, el desempeño, la reducción del ausentismo y el desarrollo de los colaboradores en la empresa [5]. El TPM se encarga de acaparar las deficiencias desde el punto más insignificante en la que se puedan encontrar, lo que demuestra que una inserción de un método como este en cualquier tipo de industria, sin importar la magnitud de la empresa, abre las puertas a una nueva fase de competitividad [6]. El TPM causa que la empresa se diferencie de su competencia gracias a que sus costos, tiempos de trabajo disminuyen considerablemente, se extiende el tiempo de vida de la planta, mejora el desarrollo de los colaboradores y mejora la calidad de los servicios [7]

\section{A. Objetivo General}

\section{OBJETIVOS}

Determinar el efecto que la aplicación de la metodología de mantenimiento productivo total tiene en el servicio de mantenimiento de molinos SAG.

\section{B. Objetivos Específicos}

- Realizar un diagnóstico de la situación actual en el servicio de mantenimiento.

- Formular propuesta de aplicación de las herramientas de Mantenimiento Productivo Total.

- Evaluación económico-financiera de la propuesta.

\section{MATERIAL Y MÉTODOS}


La presente investigación es de carácter No Experimental porque no se manipulará la variable de estudio y propositiva, porque permitirá realizar una propuesta a los servicios de mantenimiento de molinos SAG basado en el TPM. La población estará conformada por todos los procesos de mantenimiento del molino SAG y la muestra por los procesos realizados durante el periodo de estudio.

TABLA 1.

TECNICA E INSTRUMENTOS

\begin{tabular}{|c|c|c|c|c|}
\hline \begin{tabular}{|c|} 
OBJETIVO \\
ESPECÍFICO \\
\end{tabular} & FUENTE & TÉCNICA & INSTRUMENTO & LOGRO \\
\hline $\begin{array}{l}\text { Analizar y } \\
\text { diagnosticar la } \\
\text { situación actual } \\
\text { del proceso de } \\
\text { mantenimiento } \\
\text { de molinos SAG } \\
\text { que ejecuta la } \\
\text { empresa. }\end{array}$ & $\begin{array}{l}\text { Colaboradores } \\
\text { involucrados en } \\
\text { el } \\
\text { mantenimiento } \\
\text { de los molinos } \\
\text { SAG }\end{array}$ & Observación. & \begin{tabular}{lr}
\multicolumn{3}{l}{ Analizar y diagnosticar } \\
la situación actual & del \\
proceso & de \\
mantenimiento & de \\
molinos SAG & que \\
ejecuta la empresa.
\end{tabular} & $\begin{array}{l}\text { Colaboradores } \\
\text { involucrados en } \\
\text { el } \\
\text { mantenimiento } \\
\text { de los molinos } \\
\text { SAG }\end{array}$ \\
\hline $\begin{array}{l}\text { Formular la } \\
\text { propuesta de las } \\
\text { herramientas de } \\
\text { Mantenimiento } \\
\text { Productivo } \\
\text { Total a utilizar. }\end{array}$ & Colaboradores. & Encuestas & $\begin{array}{l}\text { Formular la propuesta } \\
\text { de las herramientas de } \\
\text { Mantenimiento } \\
\text { Productivo Total a } \\
\text { utilizar. }\end{array}$ & Colaboradores. \\
\hline 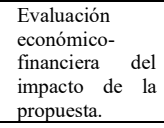 & El autor & Observación & $\begin{array}{l}\text { Evaluación económico- } \\
\text { financiera del impacto } \\
\text { de la propuesta. }\end{array}$ & El autor \\
\hline
\end{tabular}

\section{METODOLOGÍA PARA LA PROPUESTA}

Los investigadores desarrollaron la propuesta en 3 etapas las cuales consta de: Diagnóstico de la situación actual, Formulación de propuesta de aplicación y la evaluación económica-financiera.

La Tabla 2 se aprecia dichas etapas con las principales actividades.

TABLA 2

ETAPAS DE LA PROPUESTA

\begin{tabular}{|c|l|}
\hline ITEM & \multicolumn{1}{|c|}{ ETAPA } \\
\hline \multirow{1}{*}{1} & $\begin{array}{l}\text { Etapa 1: Realizar un diagnóstico de la situación actual en el servicio } \\
\text { de mantenimiento }\end{array}$ \\
& $\begin{array}{l}\text { - Determinación de las causas raíces y valor de pérdidas } \\
\text { - Descripción del impacto de las causas raíces y valor de pérdidas } \\
\text { - Monetización de Perdidas para los impactos que generan las } \\
\text { causas raíces }\end{array}$ \\
\hline \multirow{2}{*}{$\begin{array}{l}\text { Etapa 2: Formular propuesta de aplicación de las herramientas de } \\
\text { mantenimiento productivo total a utilizar para la mejora de } \\
\text { satisfacción del cliente } \\
\text { Se tomó en consideración las siguientes causa-raiz. } \\
\text { - Sensibilización y cultura de seguridad } \\
\text { - Falta de procedimientos } \\
\text { - Falta de Capacitación } \\
\text { - Falta de indicadores } \\
\text { - Falta de Herramientas de control para el mantenimiento }\end{array}$} \\
\hline $\mathbf{3}$ & Etapa 3: Evaluación económico-financiera de la propuesta \\
\hline
\end{tabular}

A. Etapa 1: Realizar un diagnóstico de la situación actual en el servicio de mantenimiento

\section{A1. DETERMINACIÓN DE LAS CAUSAS-RAICES Y VALOR DE PERDIDAS \\ Para realizar el diagnóstico del servicio de mantenimiento de Molinos SAG se utilizó el diagrama de Ishikawa para determinar e identificar la causa-raíz que originan los problemas en el mantenimiento de los molinos. El diagrama}

de Ishikawa se presenta en la Figura 1. Donde se puede apreciar el análisis de las $6 \mathrm{M}$ y las causas raíces del problema.

A continuación, se elaboró la matriz del impacto de las causas raíces, donde se priorizó las causas raíces del problema, ubicando en primer lugar a la falta de sensibilización y cultura de seguridad, seguida por la falta de procedimientos, la falta de capacitación, la falta de indicadores y la falta de herramientas de control para el mantenimiento las cuales representan el $80 \%$ de impacto en el servicio de mantenimiento. Se realizó la figura 2 dónde se aprecia que si solucionamos estas 5 causas se solucionará el $80 \%$ de problemas en el servicio de mantenimiento (Ver Figura 1).

Finalmente, se identificaron los indicadores y los valores actuales de cada una de las causas raíces y cuáles deberían ser sus indicadores para realizar la mejora en el servicio de mantenimiento de molinos SAG.

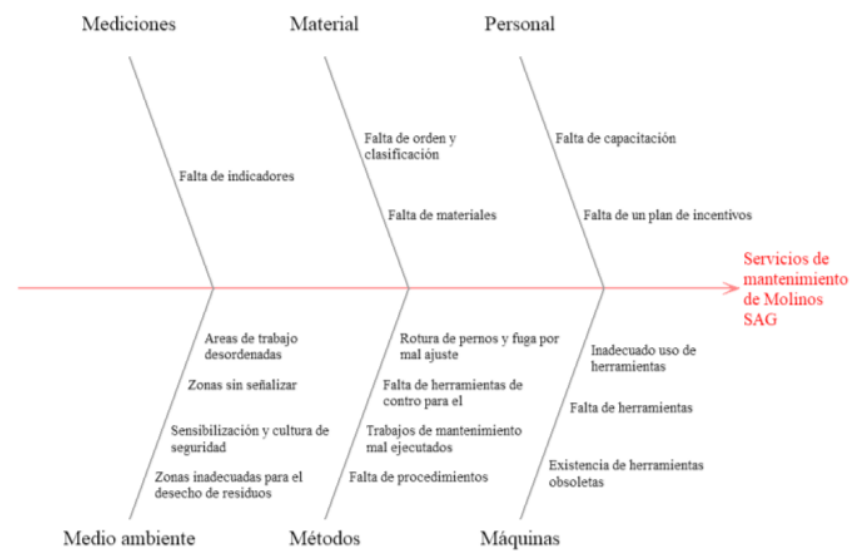

Fig.1. Diagrama de Ishikawa para el Mantenimiento de Molinos SAG. Elaboración propia

TABLA 3.

MATRIZ DEL IMPACTO DE LAS CAUSAS RAÍCES.

\begin{tabular}{|c|c|c|c|}
\hline $\begin{array}{l}\text { Causa } \\
\text { Raíz }\end{array}$ & Descripción & Impacto & $\begin{array}{c}\% \\
\text { Acumulado }\end{array}$ \\
\hline CR6 & Sensibilización y cultura de seguridad & 100 & $17 \%$ \\
\hline CR2 & Falta de procedimientos & 95 & $33 \%$ \\
\hline CR7 & Falta de capacitación & 93 & $49 \%$ \\
\hline CR1 & Falta de indicadores & 92 & $65 \%$ \\
\hline CR4 & $\begin{array}{l}\text { Falta de herramientas de control para } \\
\text { el mantenimiento }\end{array}$ & 90 & $80 \%$ \\
\hline CR10 & Áreas de trabajo desordenadas & 21 & $83 \%$ \\
\hline CR18 & Falta de materiales & 15 & $86 \%$ \\
\hline CR3 & $\begin{array}{l}\text { Trabajos de mantenimiento mal } \\
\text { ejecutados }\end{array}$ & 13 & $88 \%$ \\
\hline CR16 & $\begin{array}{l}\text { Rotura de pernos o fugas por mal } \\
\text { ajuste }\end{array}$ & 13 & $90 \%$ \\
\hline CR9 & Zonas sin señalizar & 11 & $92 \%$ \\
\hline CR11 & Inadecuado uso de los equipos & 9 & $94 \%$ \\
\hline CR5 & $\begin{array}{l}\text { Zonas inadecuadas para el desecho de } \\
\text { residuos }\end{array}$ & 8 & $95 \%$ \\
\hline CR15 & Inadecuado uso de herramientas & 7 & $96 \%$ \\
\hline CR14 & Falta de herramientas & 7 & $97 \%$ \\
\hline CR13 & Existencia de herramientas obsoletas & 5 & $98 \%$ \\
\hline CR17 & $\begin{array}{l}\text { Falta de orden y clasificación de } \\
\text { materiales }\end{array}$ & 4 & $99 \%$ \\
\hline CR8 & Falta de un plan de incentivos & 4 & $100 \%$ \\
\hline \multirow[t]{2}{*}{ CR12 } & Existencia de equipos obsoletos & 2 & $100 \%$ \\
\hline & TOTAL & 589 & \\
\hline
\end{tabular}

Elaboración propia

$1^{\text {th }}$ LACCEI International Multi-Conference for Engineering, Education, and Technology: "Industry, Innovation, And Infrastructure for Sustainable Cities and Communities", 21-23 July 2021, Buenos Aires. 


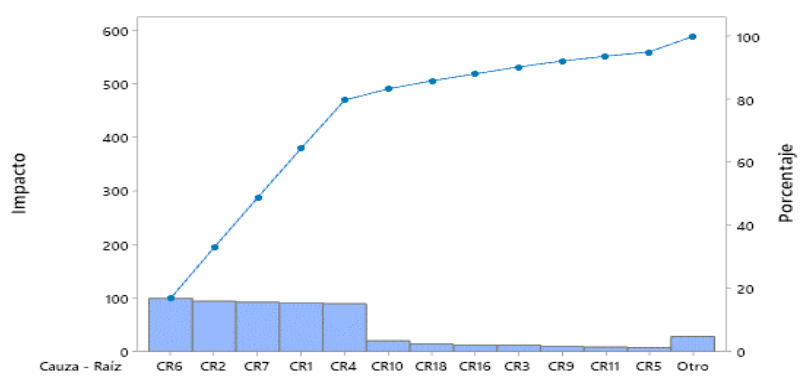

Fig.2. Diagrama de Pareto del Impacto de las Causas Raíces. Elaboración propia

\section{A2. DESCRIPCIÓN DEL IMPACTO DE LAS CAUSAS RAIICES Y VALOR DE PÉRDIDAS}

a) ICR 6: Sensibilización y cultura de seguridad

La empresa que brinda el servicio de mantenimiento de molinos SAG no cuenta con un programa de sensibilización a sus colaboradores en cuanto al tema de las prácticas de seguridad y medio ambiente al ejecutar el servicio, asimismo, no provee una zona para eliminar correctamente los residuos y los colaboradores toman la decisión de eliminar dichos residuos incorrectamente, esto es una causa de demora ya que los colaboradores tienen que volver a clasificar y a reubicar los residuos. De todo lo mencionado, se genera un ambiente de trabajo inseguro evidenciado por un área de trabajo desordenada y zonas sin señalizar correctamente, por tal condición, se considera el $60 \%$ en cuanto a sensibilización de los colaboradores.

b) ICR 2: Falta de procedimientos

La empresa no posee con un procedimiento de mantenimiento fidedigno, es por esta razón que cuando se realiza el mantenimiento, los colaboradores no saben con certeza la manera en que van a realizar el trabajo, y esa duda genera retrasos por el mal desmontaje y montaje de revestimientos, además, la empresa tiene deficiencia al realizar las actividades del mantenimiento, pues se ejecuta un mal ajuste y torqueo de los pernos ubicados en los revestimientos del molino SAG. Esta mala práctica desencadena fugas y que los revestimientos se caigan mientras el molino se encuentra operando, también se hace un mal corte de los pernos al momento del desmontaje de los revestimientos dañando a la carcasa del molino y ocasionando que los pernos no sellen el interior del molino de manera correcta.. Por tal motivo, este indicador tiene como valoración el $0 \%$.

\section{c) ICR 7: Falta de capacitación}

La empresa no cuenta con colaboradores técnicos, dado que en su mayoría son colaboradores que trabajan por un nivel de experiencia en el mantenimiento de los molinos, solamente son empíricos y por esta razón, que al momento de realizar trabajos que demanden de información técnica ellos no son capaces de realizarlo de la mejor manera, también se observa un uso inadecuado de los equipos y herramientas a utilizar, lo que genera que la ejecución de las actividades se alargue. Además, se resalta que la empresa en los dos últimos años no realizó ni un tipo de capacitación a sus colaboradores por esto, el indicador tiene como valor un $0 \%$ d) ICR 1: Falta de indicadores

La empresa no cuenta con indicadores para mapear con datos reales la manera en que se esta ejecutando los servicios de mantenimiento de molinos SAG. Este indicador está valorado con un porcentaje del $0 \%$ ya que no existe ningún indicador con el que se trabaje a la fecha.

e) ICR 4: Falta de herramientas de control para mantenimiento

No existen herramientas de control para inspección de equipos, para clasificar y ordenar los materiales de la manera adecuada, lo que causa que se opere con incertidumbre ante una falla y esto a su vez, una acumulación de demoras por falta de inspecciones en los equipos que se utilizan para ejecutar el trabajo. La empresa que solicita el servicio de mantenimiento en este caso se encarga de suministrar los materiales para la ejecución del mantenimiento, pero cuando los colaboradores están montando los revestimientos se dan con la sorpresa de que faltan pernos, tuercas y revestimientos para culminar el mantenimiento, además, el responsable solicitante del servicio no brinda las herramientas completas para realizar el mantenimiento, es por ello, que también se ocasionan demoras que extienden el tiempo de trabajo y por falta de clasificación y orden de los materiales a utilizar en el mantenimiento de molinos SAG. Por todo lo anes mencionado, este impacto tiene una valoración del $0 \%$.

\section{A3. MONETIZACIÓN DE PERDIDAS PARA LOS IMPACTOS QUE GENERAN LAS CAUSAS RAÍCES}

La falta de atención y solución sobre todos estos impactos ocasiona que el trabajo de mantenimiento de molinos SAG se realiza en un tiempo más extenso de lo que en realidad podría realizarse. En promedio el costo por día de que el cliente tenga el molino sin producir es de $\$ 125000,00$ (datos provenientes del cliente). Teniendo en cuenta que el servicio de mantenimiento se ejecuta en 8 días, el costo total es de $\$ 1000$ 000,00 .

TABLA 4.

COSTOS QUE ASUME EL CLIENTE POR TENER EL MOLINO DETENIDO PARA MANTENIMIENTO

\begin{tabular}{|c|c|}
\hline \multicolumn{2}{|l|}{ Datos Actuales } \\
\hline Costo de Molino SAG sin actividad por día & $\$ 125000,00$ \\
\hline Días de Mantenimiento de Molino SAG & 8 \\
\hline Total de costo & $\$ 1000000,00$ \\
\hline
\end{tabular}

B. Etapa 2: Formular propuesta de aplicación de las herramientas de mantenimiento productivo total a utilizar para la mejora de satisfacción del cliente

Después de haber ubicado las causas raíces y ver el impacto en el servicio de mantenimiento de molinos SAG, se plantea las propuestas de mejora para cada una de ellas, utilizando las herramientas del Mantenimiento Productivo Total.

A continuación, las propuestas para mejorar cada ICR. 
TABLA 5

PROPUESTAS DE MEJORA PARA CADA ICR

\begin{tabular}{|c|l|l|l|}
\hline ICR & \multicolumn{1}{|c|}{ Descripción } & \multicolumn{1}{|c|}{ Herramienta a utilizar } & Pilar del TPM \\
\hline ICR 6 & $\begin{array}{l}\text { Sensibilización y } \\
\text { cultura de } \\
\text { seguridad }\end{array}$ & $\begin{array}{l}\text { Programa de } \\
\text { sensibilización } \\
\text { 5s }\end{array}$ & $\begin{array}{l}\text { Seguridad y } \\
\text { Medio Ambiente }\end{array}$ \\
\hline ICR 2 & $\begin{array}{l}\text { Falta de } \\
\text { procedimientos } \\
\text { procedimientos para el } \\
\text { desarrollo del servicio de } \\
\text { mantenimiento de } \\
\text { molinos SAG } \\
\text { Elaboración de Gantt }\end{array}$ & $\begin{array}{l}\text { Mantenimiento } \\
\text { Planificado }\end{array}$ \\
\hline ICR 1 & $\begin{array}{l}\text { Falta de } \\
\text { Capacitación } \\
\text { indicadores de }\end{array}$ & $\begin{array}{l}\text { Programas de } \\
\text { Capacitaciones }\end{array}$ & $\begin{array}{l}\text { Educación } \\
\text { Entrenamiento }\end{array}$ \\
\hline ICR 4 de & $\begin{array}{l}\text { Herramientas de } \\
\text { control para el } \\
\text { mantenimiento }\end{array}$ & $\begin{array}{l}\text { Mejoras } \\
\text { Hoja de inspección de los } \\
\text { equipos y herramientas }\end{array}$ & $\begin{array}{l}\text { Mantenimiento } \\
\text { Autónomo }\end{array}$ \\
\hline
\end{tabular}

Elaboración propia

A) PARA ICR 6: Sensibilización y Cultura de Seguridad

La empresa que brinda el servicio de mantenimiento de molinos SAG no tiene sensibilizados a sus colaboradores en cuanto al tema de las prácticas de seguridad y medio ambiente al ejecutar el servicio. Asimismo, no provee una zona para eliminar correctamente los residuos y los colaboradores toman la decisión de eliminarlos en cualquier lugar donde se encuentre despejado. De la misma maner, no cuentan con un programa de sensibilización para sus colaboradores evidenciado por un área de trabajo desordenada y zonas sin señalizar correctamente.

Por ende, se propone desarrollar una programación de charlas para sensibilizar a los colaboradores en cuanto a trabajar de manera segura y cuidando al medio ambiente; además de esto se aplicará la herramienta $5 \mathrm{~s}$ para fomentar una cultura de orden y limpieza en el área de trabajo.

A continuación, se proponen las siguientes actividades para el programa de Seguridad y Medio Ambiente.

$$
\text { TABLA } 6
$$

ACTIVIDADES DEL PROGRAMA DE SEGURIDAD Y MEDIO

\begin{tabular}{|c|c|}
\hline OBJETIVOS & ACTIVIDADES \\
\hline $\begin{array}{l}\text { 1. Mejora del Sistema de } \\
\text { Gestión de Seguridad y } \\
\text { Salud en el Trabajo }\end{array}$ & $\begin{array}{l}\text { - Difusión de política de seguridad y salud en } \\
\text { el trabajo (Semestral) } \\
\text { - Revisión y Difusión del reglamento de } \\
\text { seguridad, salud en el trabajo (Semestral) }\end{array}$ \\
\hline 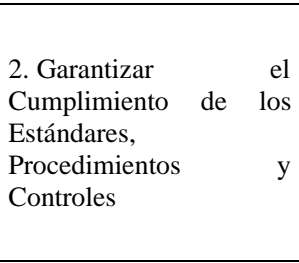 & $\begin{array}{l}\text { - } \quad \text { Supervisión permanente de los trabajos en el } \\
\text { servicio de mantenimiento } \\
\text { - } \quad \text { Auditorías internas en el tema de seguridad } \\
\text { y salud en el trabajo } \\
\text { - } \quad \text { Difusión de procedimientos } \\
\text { - } \quad \text { Simulacros de primeros auxilios } \\
\text { - } \quad \text { Inspección de la selección y eliminación de } \\
\text { los residuos }\end{array}$ \\
\hline $\begin{array}{lr}\text { 3. Seguimiento } & \text { del } \\
\text { Cronograma de Charlas, } \\
\text { Concientización } & \text { y } \\
\text { Desarrollo de } \\
\text { Competencias } & \text { en } \\
\text { Seguridad y medio } \\
\text { ambiente. }\end{array}$ & $\begin{array}{l}\text { - Difusión de cuadro de obligaciones y } \\
\text { sanciones (semestral) } \\
\text { - Inducción de ingreso a los colaboradores } \\
\text { nuevos } \\
\text { - Monitoreo de calidad de aire y nivel de ruido } \\
\text { en planta } \\
\text { - } \quad \text { Evaluación ergonómica y de iluminación }\end{array}$ \\
\hline
\end{tabular}

Estas actividades se deben desarrollar en todos los servicios de mantenimiento, con el fin de fortalecer la prevención de accidentes y enfermedades ocupacionales, así como el impacto al medio ambiente.

\section{IMPLEMENTACIÓN DE LA METODOLOGÍA 5S}

\section{a) Etapa 1: Seiri - Clasificar}

En esta etapa los colaboradores tienen como tarea principal clasificar todas las máquinas, herramientas, componentes e insumos existentes en el área de trabajo separando lo que es de uso seguro de lo inseguro; lo identificado como inseguro deberá ser etiquetado con una tarjeta amarilla de fuera de servicio que se presenta líneas abajo, en esta se coloca la descripción del equipo o herramienta, nombre, área y fecha, estos objetos etiquetados con la tarjeta amarilla deberán ser contabilizados para su posterior análisis.
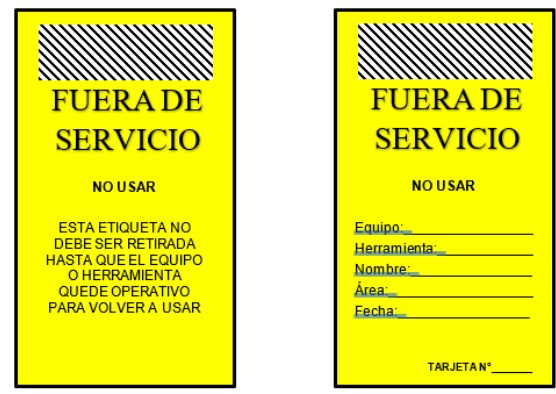

Fig.3. Tarjeta amarilla de identificación de equipos o herramientas inseguras Elaboración Propia Minitab 2019.

\section{b) Etapa 2: Seiton - Ordenar}

Luego de clasificar los equipos y herramientas en el área de trabajo se sigue con el orden y ubicación en lugares de fácil acceso y para aquellos equipos o herramientas inseguras se les dará un tratamiento para ver si cambia a operativo o se desecha en caso de que se evidencie que se encuentran obsoletos.

Las actividades a seguir durante esta etapa se detallan a continuación en la Tabla 7.

TABLA 7

CRITERIOS DE ORDENAMIENTO DE LA METODOLOGÍA 5S

\begin{tabular}{|c|c|c|}
\hline Área & Criterios & Responsable \\
\hline Planta & $\begin{array}{l}\text { - Delimitar el área. } \\
\text { - Señalizar con cartillas visibles. } \\
\text { - Ordenar herramientas de acuerdo a } \\
\text { clasificación interna. } \\
\text { - Realizar inventario de equipos y } \\
\text { herramientas. }\end{array}$ & $\begin{array}{l}\text { - Técnicos mecánicos. } \\
\text { - Lideres. } \\
\text { - Supervisor mecánico. } \\
\text { - Supervisor de seguridad } \\
\text { y medio ambiente. }\end{array}$ \\
\hline Oficina & Ordenar el área adecuadamente. & $\begin{array}{ll}\text { - } & \text { Colaboradores } \\
& \text { administrativos. }\end{array}$ \\
\hline
\end{tabular}

Estos campos deben contener la siguiente información:

Ubicación: Se refiere al código de compartimiento donde está ubicado, esto permitirá que las herramientas, materiales y equipos sean ubicadas de manera más rápida.

Detalle: Es el nombre con el que se identifica la herramienta.

Existencias: indica la cantidad de herramientas, materiales y equipos disponibles.

Estado: indica la condición de la existencia, nuevo o usado.

Código: se refiere a la identificación del equipo.

$19^{\text {th }}$ LACCEI International Multi-Conference for Engineering, Education, and Technology: "Industry, Innovation, And Infrastructure for Sustainable Cities and Communities", 21-23 July 2021, Buenos Aires. 
Comentario: indica alerta por falta de alguna existencia o daños encontrados, por lo cual se debe coordinar una solución inmediata.

Terminadas las actividades de esta etapa, se procede a realizar una inspección por parte del equipo responsable de la implementación del programa de mantenimiento productivo total, los cuales darán paso para el inicio de la siguiente etapa.

\section{c) Etapa 3: Seiso - Limpiar}

Terminadas las anteriores etapas, se procede con una limpieza exhaustiva de herramientas, equipos, materiales y del área de trabajo. Esta etapa es considerada como una actividad de inspección que sirve para cerciorarnos de que las etapas anteriores de clasificación y ordenamiento se ejecutarón de manera correcta. Las actividades a considerar se muestran en la Tabla 8 .

TABLA 8

ACTIVIDADES DE ETAPA DE LIMPIEZA.

\begin{tabular}{|c|c|c|}
\hline Área & Criterios & Responsable \\
\hline Planta & $\begin{array}{ll}\text { - } & \text { Limpieza de herramientas, } \\
\text { equipos y materiales. } \\
\text { - } \quad \text { Limpieza del área de trabajo. } \\
\text { - } \quad \text { Inspección del área de trabajo. }\end{array}$ & $\begin{array}{l}\text { - Técnicos mecánicos } \\
\text { - Líderes } \\
\text { - Supervisor mecánico } \\
\text { - Supervisor de seguridad } \\
\text { - y medio ambiente }\end{array}$ \\
\hline Oficina & $\begin{array}{l}\text { - Limpieza de equipos y } \\
\text { herramientas. } \\
\text { - Limpieza del área de trabajo. } \\
\text { - Inspección final. }\end{array}$ & $\begin{array}{ll}\text { - } & \text { Colaboradores } \\
\text { administrativos }\end{array}$ \\
\hline
\end{tabular}

Elaboración propia

d) Etapa 4: Seiketsu - Estandarizar

Esta etapa tiene como objetivo conservar y practicar interrumpidamente los buenos hábitos de orden y limpieza en las áreas de trabajo, para lo cual, se propone establecer procedimientos para su cumplimiento obligatorio por responsables de cada área. En la Tabla 9 se ha desarrollado la secuencia de actividades del proceso del servicio de mantenimiento cuyo cumplimiento es obligatorio por todos los involucrados.

TABLA 9

SECUENCIA DE ACTIVIDADES DEL PROCESO DEL SERVICIO DE

\begin{tabular}{|c|c|c|c|c|}
\hline \multicolumn{5}{|c|}{$D_{0}$} \\
\hline $\mathbf{v}$ & $\begin{array}{c}\text { Activida } \\
\mathrm{d}\end{array}$ & Documento & Descripcion & Kesponsabie \\
\hline 1 & $\begin{array}{l}\text { Recepci } \\
\text { ón }\end{array}$ & $\begin{array}{l}\text { - Registro de } \\
\text { recepción de } \\
\text { molino } \\
\text { SAG }\end{array}$ & $\begin{array}{l}\text { - Condiciones de } \\
\text { recepción del molino y } \\
\text { el área }\end{array}$ & $\begin{array}{l}\text { Jefe de } \\
\text { Mantenimiento. } \\
\text { Supervisor } \\
\text { Mecánico.. }\end{array}$ \\
\hline 2 & $\begin{array}{l}\text { Preparac } \\
\text { ión }\end{array}$ & $\begin{array}{l}\text { - Check list de } \\
\text { herramientas } \\
\text {, equipos y } \\
\text { materiales }\end{array}$ & $\begin{array}{l}\text { - Revisión de } \\
\text { herramientas, equipos } \\
\text { y materiales a utilizar } \\
\text { para el servicio de } \\
\text { mantenimiento }\end{array}$ & $\begin{array}{l}\text { Supervisor } \\
\text { Mecánico. }\end{array}$ \\
\hline 3 & $\begin{array}{l}\text { Manteni } \\
\text { miento }\end{array}$ & $\begin{array}{c}\text { Manual de } \\
\text { molino }\end{array}$ & 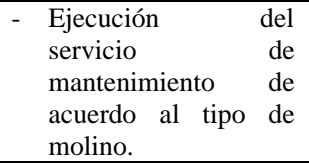 & $\begin{array}{l}\text { Técnico } \\
\text { Mecánico. } \\
\text { Líder. } \\
\text { Supervisor } \\
\text { Mecánico. }\end{array}$ \\
\hline 4 & $\begin{array}{l}\text { Inspecci } \\
\text { ón Final }\end{array}$ & $\begin{array}{l}\text { Hoja de } \\
\text { verificación }\end{array}$ & $\begin{array}{lr}\text { Control de calidad } \\
\text { del servicio de } \\
\text { mantenimiento } & \text { de } \\
\text { acuerdo a } & \text { los } \\
\text { estándares } & \text { de } \\
\text { seguridad } & \\
\text { establecidos } & \\
\end{array}$ & $\begin{array}{l}\text { Supervisor } \\
\text { Mecánico. } \\
\text { Supervisor de } \\
\text { Seguridad y } \\
\text { medio ambiente. } \\
\text { Jefe de } \\
\text { Mantenimiento. }\end{array}$ \\
\hline
\end{tabular}

e) Etapa 5: Shitsuke - Disciplina

Esta etapa se encarga de concientizar a los colaboradores para que mantengan el orden, limpieza y las buenas prácticas de trabajo en cada puesto de trabajo que permitirá reducir los incidentes y accidentes en el área y así cumplir con las metas del cliente y a la misma vez de la empresa que brida el servicio de mantenimiento de molinos SAG.

\section{PLAN DE MANTENIMIENTO DE MOLINO SAG}

a) Al iniciar el servicio de mantenimiento:

- Utilizar el uniforme y EPPs adecuadamente.

- Planificar actividades a ejecutar

- Identificar los peligros y riesgos en el área de trabajo.

b) Durante el Mantenimiento:

- Alistar y organizar las herramientas, equipos y materiales a utilizar.

- Seguir el proceso de mantenimiento.

- Utilizar las herramientas, equipos y materiales adecuados para la ejecución del mantenimiento.

- Utilizar constantemente el EPP.

- Mantener el orden y limpieza.

c) Al Finalizar el Servicio de Mantenimiento:

- Re ubicar cada herramienta, equipo a su lugar.

- Limpiar el área de trabajo.

- Verificar que todo quede en perfecto estado para volver a poner en funcionamiento el molino SAG.

Si los colaboradores continúan con las malas prácticas de trabajo y no muestra interés por el cambio, se procederá a escuchar su versión para poder aclarar los puntos y así apoyarlo y tener como resultado que no se vuelva a presentar los mismos errores en ningún colaborador involucrado en el servicio de mantenimiento.

B) PARA ICR 2: Falta de Procedimientos.

La empresa no posee con un procedimiento de mantenimiento fidedigno, es por esta razón, que cuando se realiza el mantenimiento, los colaboradores no saben con certeza la manera en que van a realizar el trabajo y esa duda genera retrasos por el mal desmontaje y montaje de revestimientos, además la empresa tiene deficiencia al realizar las actividades del mantenimiento, ejecuta un mal ajuste y torqueo de los pernos ubicados en los revestimientos del molino SAG, esta mala práctica desencadena fugas y que los revestimientos se caigan mientras el molino se encuentra operando, también se hace un mal corte de los pernos al momento del desmontaje de los revestimientos dañando a la carcasa o shell del molino y ocasionando que los pernos no sellen el interior del molino de manera correcta.

DESARROLLO DE LA PROPUESTA DE MEJORA

Se propone elaborar un procedimiento de trabajo para el servicio de mantenimiento.

Se propone elaborar un diagrama Gannt para que los colaboradores tengan una referencia y recordatorio de como se desarrolla el servicio de mantenimiento.

$1^{\text {th }}$ LACCEI International Multi-Conference for Engineering, Education, and Technology: "Industry, Innovation, And Infrastructure for Sustainable Cities and Communities", 21-23 July 2021, Buenos Aires. 


\section{C) PARA ICR 7:}

La empresa no cuenta con colaboradores técnicos, ya que en su mayoría son colaboradores que trabajan por un nivel de experiencia en el mantenimiento de los molinos, solamente son empíricos y por esta razón que al momento de realizar trabajos que demanden de información técnica ellos no son capaces de realizarlo de la mejor manera, también se observa un uso inadecuado de los equipos y herramientas a utilizar lo que también genera que la ejecución de las actividades se alargue.

Para dar un servicio que supere las expectativas del cliente y así conseguir su satisfacción, es necesario brindar capacitaciones que presenten temas que potencie el conocimiento técnico de los colaboradores para realizar el servicio de mantenimiento. Para desarrollar esta mejora se propone aplicar el siguiente pilar del TPM.

Quinto Pilar: Educación y Entrenamiento

se necesita la participación activa de todos los colaboradores involucrados para así desarrollar las competencias de los colaboradores, teniendo en cuenta los objetivos de la empresa.

Este programa de capacitación consta de 5 capacitaciones que se realizaran a lo largo de lo que resta del año 2020 y 2021.

A continuación, en la Figura 4 se muestra el cronograma para las capacitaciones propuestas.

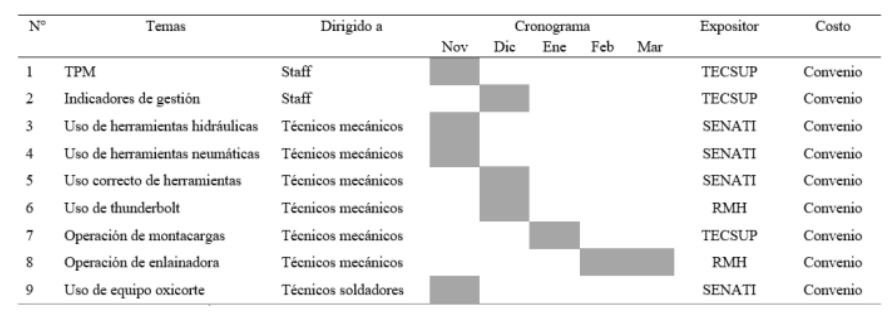

Fig.4. Cronograma de Charlas propuestas a técnicos.

Elaboración propia

D) PARA ICR 1: Falta de Indicadores

La empresa no cuenta con indicadores para mapear con datos reales la manera en que se está ejecutando los servicios de mantenimiento de molinos SAG.

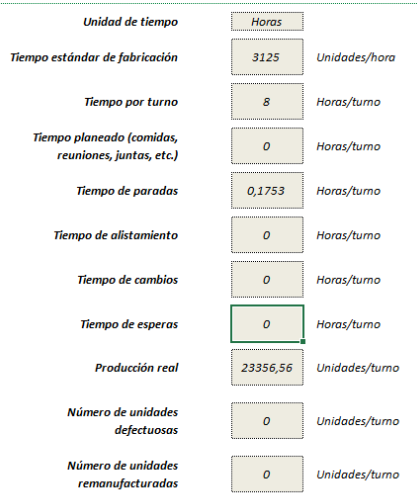

Fig.5. Datos necesarios para el cálculo de los indicadores OEE. Elaboración propia

En la Figura 4 podemos apreciar los datos necesarios para los cálculos de los indicadores, se debe tener en cuenta las unidades de tiempo, el tiempo estándar, los tiempos por turno, los tiempos perdidos, la producción real, las unidades defectuosas. Los datos ingresados corresponden al proceso de mantenimiento de molinos SAG.

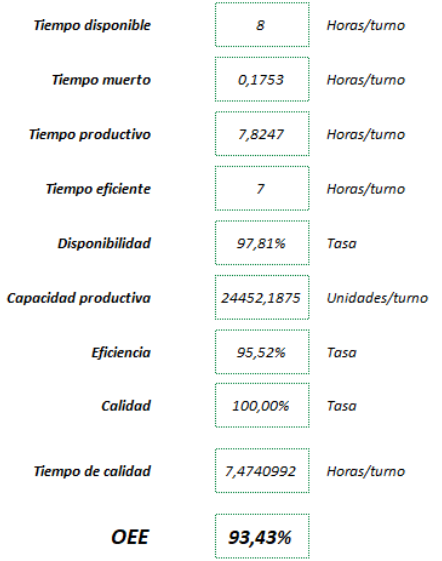

Fig.6. Cálculos obtenidos con las fórmulas de OEE. Elaboración propia

En la Figura 6 se puede apreciar los resultados obtenidos con los datos de la Figura 5, aquí con las fórmulas de los indicadores OEE se hallan los valores de Disponibilidad, Eficiencia y Calidad. Y así con estos datos obtener el OEE del proceso de mantenimiento de molinos SAG que asciende a $93,43 \%$ que, si bien es un buen indicador, este se podría mejorar aún más con la implementación del TPM.

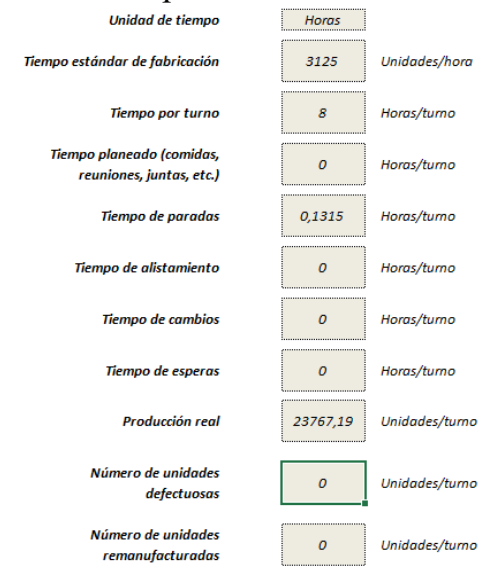

Fig.7. Datos necesarios para el cálculo de los indicadores OEE. Elaboración propia

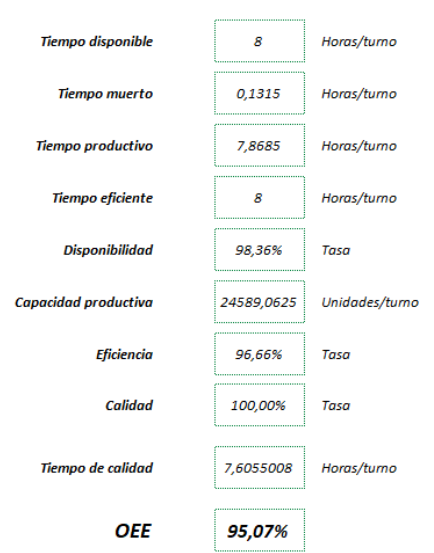

Fig.8. Cálculos obtenidos con las fórmulas de OEE con la mejora. Elaboración propia

$19^{\text {th }}$ LACCEI International Multi-Conference for Engineering, Education, and Technology: "Industry, Innovation, And Infrastructure for Sustainable Cities and Communities", 21-23 July 2021, Buenos Aires. 
E) PARA ICR 4: Falta de Herramientas de Control

No existe documentación para inspección de equipos, para clasificar y ordenar los materiales de la manera adecuada, lo que causa que se opere con incertidumbre ante una falla y esto cause una vez más una acumulación de demoras por falta de inspecciones en los equipos que se utilizan para ejecutar el trabajo. La empresa que solicita el servicio de mantenimiento en este caso se encarga de suministrar los materiales para la ejecución del mantenimiento, pero cuando los colaboradores están montando los revestimientos se dan con la sorpresa de que faltan pernos, tuercas y revestimientos para culminar el mantenimiento, además la empresa solicitante del servicio no brinda las herramientas completas para realizar el mantenimiento, es por esto que también se ocasionan demoras que extienden el tiempo de trabajo.

Los formatos que se proponen a continuación son:

- Formato para ubicación de liners en plataforma.

- Formato para requerimiento de herramientas

- Formato para requerimiento de materiales

- Formato para requerimiento de Equipos

- Matriz para torque de pernos y tuercas

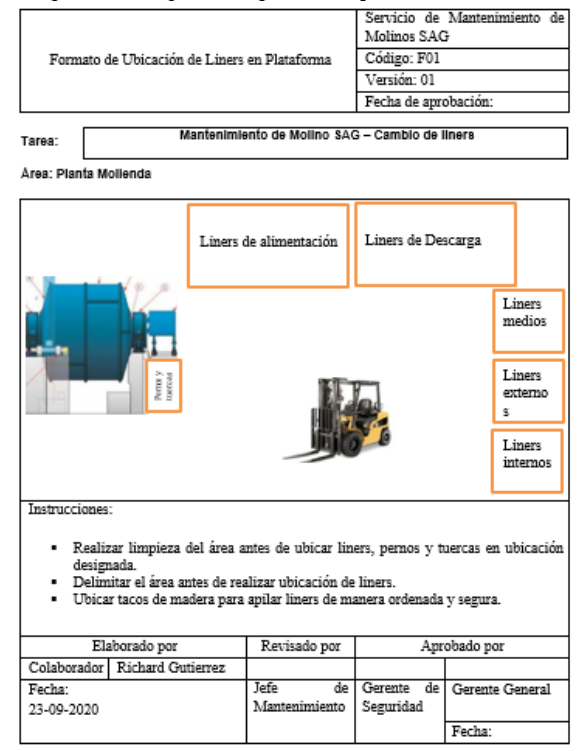

Fig.9. Formato para ubicación de liners en plataforma Elaboración propia

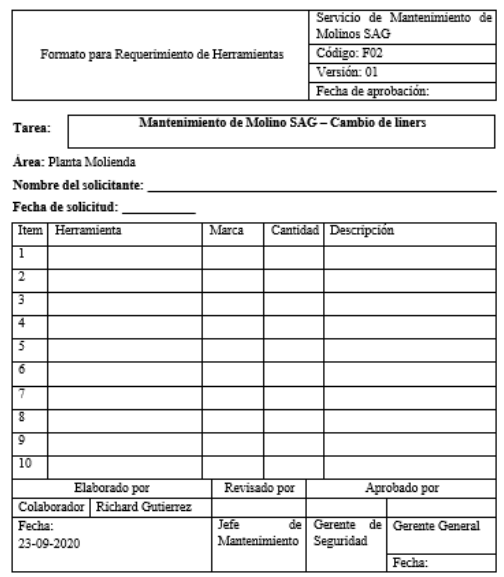

Fig.10. Formato para requerimiento de herramientas. Elaboración propia

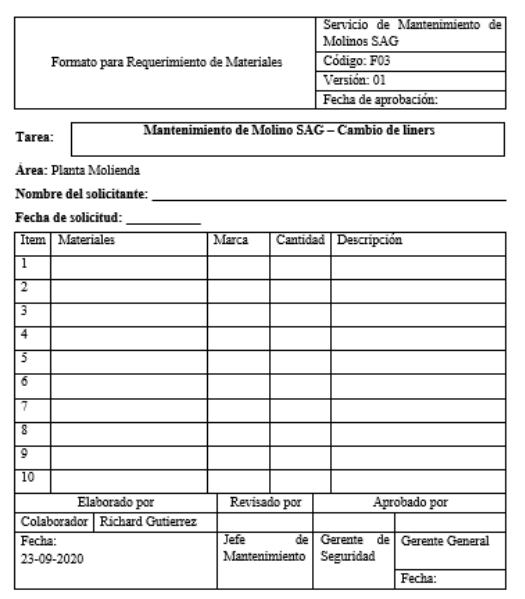

Fig.11. Formato para requerimiento de materiales. Elaboración propia

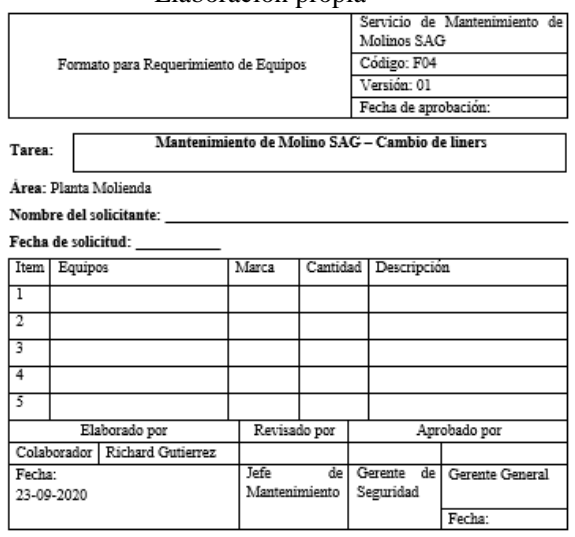

Fig.12. Formato para requerimiento de Equipos. Elaboración propia
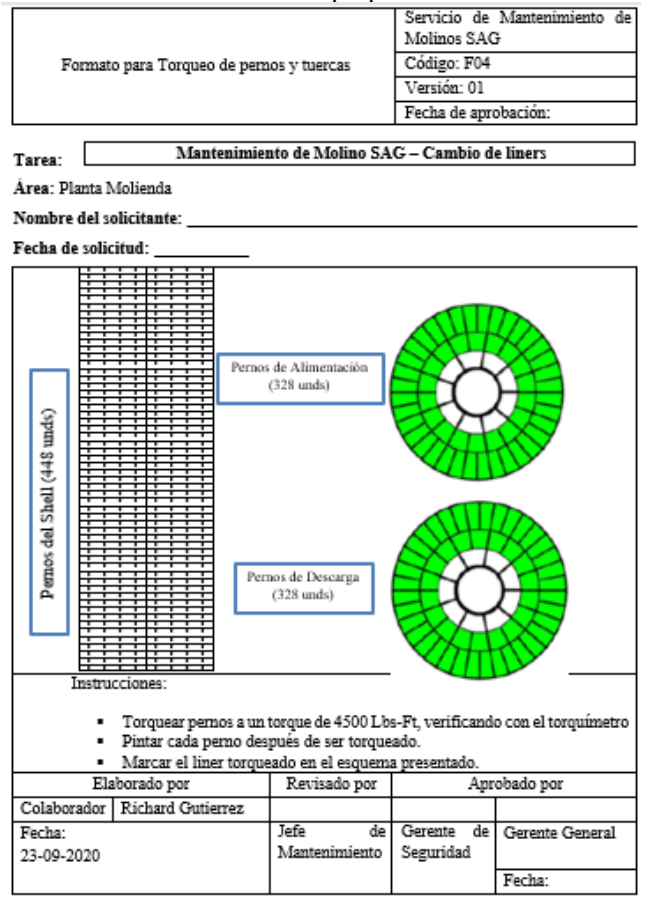

Fig.13. Matriz para torque de pernos y tuercas. Elaboración propia

$19^{\text {th }}$ LACCEI International Multi-Conference for Engineering, Education, and Technology: "Industry, Innovation, And Infrastructure for Sustainable Cities and Communities", 21-23 July 2021, Buenos Aires. 
C. Etapa 3: Evaluación económico-financiera de la propuesta

La evaluación económica de la propuesta de mejora tendría una inversión en S/67 500,00 que incluye capacitaciones a cargo de Senati y Tecsup, la implementación de las 5S, la compra de una PC de escritorio y una impresora láser. Se complementa con la compra de mobiliario para el área de Control de TPM.

TABLA 10

INVERSIÓN DE LA PROPUESTA DE MEJORA AL SERVICIO DE

MANTENIMIENTO DE MOLINOS SAG
\begin{tabular}{|c|c|}
\hline Descripción & Monto (S/) \\
\hline Capacitaciones Senati & $\mathrm{S} / 21000,00$ \\
\hline Capacitaciones Tecsup & $\mathrm{S} / 30000,00$ \\
\hline Implementación 5S & $\mathrm{S} / 8500,00$ \\
\hline Computadora & $\mathrm{S} / 3500,00$ \\
\hline Impresora & $\mathrm{S} / 1000,00$ \\
\hline Mobiliario & $\mathrm{S} / 3500,00$ \\
\hline Total & $\mathrm{S} / 67500,00$ \\
\hline
\end{tabular}

Elaboración propia

Los ingresos anuales de la propuesta de mejora están dados por el ahorro que se realizará al momento de efectuar el mantenimiento de los molinos SAG siguiendo las recomendaciones y proceso estandarizado de la propuesta. Con lo cual reduciremos en dos días el mantenimiento generando un ahorro de US\$ 100 000,00, dicho calculo esta establecido por el área de contabilidad de la empresa. El tipo de cambio se ha considerado de 3,50 soles por dólar.

TABLA 11

INGRESOS ANUALES DE LA PROPUESTA DE MEJORA AL SERVICIO DE MANTENIMIENTO DE MOLINOS SAG

\begin{tabular}{|c|r|}
\hline Ingresos & Monto (S/) \\
\hline Reducción del tiempo de mantenimiento (2 días) & $\mathrm{S} / 350000,00$ \\
\hline Total & $\mathrm{S} / 350000,00$ \\
\hline \multicolumn{2}{|c|}{ Elaboración propia }
\end{tabular}

Los egresos anuales se darán por la contratación de un Ingeniero Industrial para el control y monitoreo de los indicadores TPM con un sueldo de 3 400,00 soles más sus beneficios sociales (EsSalud, SCRT, Asignación Familiar, Vacaciones, CTS, etc.) y los útiles de escritorio por un monto mensual de 125,00 soles, para asegurar los beneficios que se pretenden alcanzar con la propuesta de mejora (Ver Anexo 4). TABLA 12

EGRESOS ANUALES DE LA PROPUESTA DE MEJORA AL SERVICIO DE MANTENIMIENTO DE MOLINOS SAG

\begin{tabular}{|c|l|}
\hline \multicolumn{1}{|c|}{ Egresos } & \multicolumn{1}{|c|}{ Monto (S/) } \\
\hline Personal contratado para el monitoreo de TPM & S/56 202,37 \\
\hline Útiles escritorio & S/1 500,00 \\
\hline Total & S/57 702,37 \\
\hline Elaboración propia
\end{tabular}

Toda esta información ha generado el siguiente flujo de efectivo que se muestra en la siguiente tabla:

TABLA 13

FLUJO ECONÓMICO DE LA PROPUESTA DE MEJORA AL SERVICIO DE MANTENIMIENTO DE MOLINOS SAG

\begin{tabular}{|c|c|c|c|c|}
\hline & 2020 & \multicolumn{1}{|c|}{2021} & 2022 & 2023 \\
\hline Ingresos & & $\mathrm{S} / 350000,00$ & $\mathrm{~S} / 350000,00$ & $\mathrm{~S} / 350000,00$ \\
\hline Inversión & $\mathrm{S} / 67500,00$ & & & \\
\hline Egresos & & $\mathrm{S} / 57702,37$ & $\mathrm{~S} / 57702,37$ & $\mathrm{~S} / 57702,37$ \\
\hline Flujo Neto & $-\mathrm{S} / 67500,00$ & $\mathrm{~S} / 292297,63$ & $\mathrm{~S} / 292297,63$ & $\mathrm{~S} / 292297,63$ \\
\hline \multicolumn{5}{|c|}{ Elaboración propia }
\end{tabular}

La evaluación económica a este flujo de efectivo considerando una tasa de interés del $15 \%$ es:

$\mathrm{VAN}=\mathrm{S} / 599881,29$

$\mathrm{TIR}=430 \%$

Lo que indica que nuestra propuesta de mejora en el servicio de mantenimiento de molinos SAG es factible $\mathrm{y}$ genera rentabilidad.

VI. RESULTADOS

TABLA 14

RESULTADOS

\begin{tabular}{|c|c|c|c|c|c|c|c|c|c|c|c|}
\hline $\mathbf{N}^{\circ}$ & Causa Raíz & Indicador & Fórmula & Escala & $\begin{array}{l}\text { Valor } \\
\text { Actual }\end{array}$ & $\begin{array}{l}\text { Pérdida } \\
\text { (S.) }\end{array}$ & $\begin{array}{l}\text { Valor } \\
\text { ropuesto }\end{array}$ & $\begin{array}{c}\text { Pérdida } \\
\text { (dismin } \\
\text { uida) }\end{array}$ & $\begin{array}{c}\text { Beneficio } \\
\text { propuesto } \\
(\$) \\
\end{array}$ & $\begin{array}{c}\text { Herramienta } \\
\text { propuesta }\end{array}$ & $\begin{array}{l}\text { Metodología } \\
\text { Propuesta }\end{array}$ \\
\hline $\begin{array}{c}\text { ICR } \\
6\end{array}$ & $\begin{array}{l}\text { Sensibilización y } \\
\text { cultura } \\
\text { seguridad }\end{array}$ & $\begin{array}{l}\text { Frecuencia de } \\
\text { accidentes }\end{array}$ & $\frac{\text { Frecuencia de accidentes }}{\text { HH trabajadas }} \times 100$ & $\%$ & $60 \%$ & \multirow{5}{*}{$\begin{array}{l}\$ 1000 \\
000,00\end{array}$} & 0 & \multirow{5}{*}{$\begin{array}{c}\$ 900 \\
000,00\end{array}$} & \multirow{5}{*}{$\$ 100000,00$} & $\begin{array}{l}6^{\circ} \text { PILAR TPM: } \\
\text { Seguridad y Medio } \\
\text { Ambiente. }\end{array}$ & \multirow{5}{*}{$\begin{array}{l}\text { Mantenimiento } \\
\text { Productivo Total }\end{array}$} \\
\hline $\begin{array}{c}\text { ICR } \\
2\end{array}$ & $\begin{array}{l}\text { Falta de } \\
\text { procedimientos }\end{array}$ & $\begin{array}{l}\text { \%de } \\
\text { procedimientos } \\
\text { para trabajos } \\
\text { realizados }\end{array}$ & $\frac{\text { Procedimiento de trabajos }}{\text { Total de etapas del mantto. }} \times 100$ & $\%$ & $0 \%$ & & $90 \%$ & & & $\begin{array}{l}3^{\circ} \text { PILAR TPM: } \\
\text { Mantenimiento } \\
\text { planificado. }\end{array}$ & \\
\hline $\begin{array}{c}\text { ICR } \\
7\end{array}$ & $\begin{array}{l}\text { Falta } \\
\text { Capacitación }\end{array}$ & $\begin{array}{l}\% \\
\text { colaboradores } \\
\text { capacitados }\end{array}$ & $\frac{N^{\circ} \text { colaboradores capacitados }}{\text { Total de colaboradores }} \times 100$ & $\%$ & $0 \%$ & & $100 \%$ & & & $\begin{array}{l}5^{\circ} \text { PILAR TPM: } \\
\text { Educación y } \\
\text { Entrenamiento. }\end{array}$ & \\
\hline $\begin{array}{c}\text { ICR } \\
1\end{array}$ & $\begin{array}{l}\text { Falta } \\
\text { indicadores }\end{array}$ & $\begin{array}{l}\% \text { de indicadores } \\
\text { para el } \\
\text { mantenimiento de } \\
\text { molinos SAG }\end{array}$ & $\frac{\text { Indicadores }}{\text { Total de impacto de causus raices }} \times 100$ & $\%$ & $0 \%$ & & $90 \%$ & & & $\begin{array}{l}1^{\circ} \text { PILAR TPM: } \\
\text { Mejoras Enfocadas }\end{array}$ & \\
\hline $\begin{array}{c}\text { ICR } \\
4\end{array}$ & $\begin{array}{ll}\text { Falta } & \text { de } \\
\text { Herramientas } & \text { de } \\
\text { control para el } \\
\text { mantenimiento }\end{array}$ & $\begin{array}{ll}\% & \mathrm{de} \\
\text { Herramientas } & \mathrm{de} \\
\text { control para el } \\
\text { mantenimiento de } \\
\text { molinos SAG. }\end{array}$ & $\frac{\text { Herramientas de contral }}{\text { Total de herramientas para elmant to. }} \times 100$ & $\%$ & $0 \%$ & & $90 \%$ & & & $\begin{array}{l}2^{\circ} \text { PILAR TPM: } \\
\text { Mantenimiento } \\
\text { autónomo. }\end{array}$ & \\
\hline
\end{tabular}

Elaboración propia

$1^{\text {th }}$ LACCEI International Multi-Conference for Engineering, Education, and Technology: "Industry, Innovation, And Infrastructure for Sustainable Cities and Communities”, 21-23 July 2021, Buenos Aires. 


\section{DISCUSIONES}

Se logró realizar el diagnóstico de la situación actual del servicio de mantenimiento de molinos SAG a través de las herramientas de Diagrama de Ishikawa, Matriz de Priorización y Diagrama de Pareto, ubicando 5 causas raíces -Sensibilización y cultura de seguridad, Falta de procedimientos, Falta de capacitación, Falta de indicadores y Falta de herramientas de control para el mantenimiento- que son las que originan el $80 \%$ de problemas en el servicio como se aprecia en las Figuras 1 y 2, y la Tabla 2. Esto coincide con Aguilar (2018) quien manifiesta en su investigación que las mejoras planteadas atacan las principales causas como la implementación de maquinaria, elaboración de procedimientos, capacitación del personal y el trabajo con los proveedores sobre el diseño y mejora de la maquinaria, así como la medición de la fiabilidad y efíciencia de los equipos.

En la investigación se presenta la situación actual e indicadores, y asimismo los indicadores propuestos con la mejora del TPM. Para lograr estos objetivos se plantean capacitaciones a todo el personal involucrado en el servicio de mantenimiento de molinos SAG, Tablas 6 y 12, por parte de instituciones como Senati y Tecsup líderes en capacitación tecnológica. Asimismo, se plantea una estandarización de los procesos de mantenimiento de los molinos SAG y la reducción de los tiempos de mantenimiento como se muestra en la Tabla 10 y 11. Coincidimos con García (2018) quien concluye que la innovación tecnológica en cuanto a los procesos industriales siempre tiene que ir de la mano de una adecuada gestión de planta y poder establecer metas que ayuden a tener mejores resultados y sobre todo que genere una mayor satisfacción por parte de los colaboradores.

La evaluación económica de la propuesta de mejora tendría una inversión en S/67 500,00 (Tabla 14) que incluye capacitaciones a cargo de Senati y Tecsup, la implementación de las 5S, la compra de una PC de escritorio y una impresora láser. Se complementa con la compra de mobiliario para el área de Control de TPM. Asimismo, los ahorros en mantenimiento vendrían a ser nuestros ingresos anuales los cuales ascienden a $\mathrm{S} / 350$ 000,00 (Tabla 15) y los egresos anuales ascenderían a $\mathrm{S} / 57500,00$ entre personal y útiles de escritorio (Tabla 16). El análisis de los indicadores de evaluación utilizando una tasa de descuento de $15 \%$ nos da un VAN de S/600 343,35 y una TIR de $430 \%$. Se coincide con Llontop (2018) quien indica que con apoyo mediante el mantenimiento autónomo se llega a un $75 \%$ de efectividad, lo cual ayuda en la productividad para mayor reducción de tiempo a la disponibilidad que es el punto más indispensable que mejorar y por otro lado Acuña (2019) indica que implementar los pilares del TPM en el área de producción dio como resultado un impacto fuerte en la reducción de los costos de mantenimiento y horas de paradas por mantenimiento correctivo y por lo tanto ayuda a que las maquinarias tengan un flujo de trabajo constante.

\section{CONCLUSIONES}

Se concluye que la aplicación de la metodología de TPM tuvo un efecto positivo en el servicio de mantenimiento de molinos SAG dado que genera un ahorro de S/350 000,00 anuales en el servicio.

Se logró realizar el diagnóstico del servicio de mantenimiento de molinos SAG utilizando el diagrama de Ishikawa, la matriz de priorización y el diagrama de Pareto que permitieron encontrar las causas raíces -Sensibilización y cultura de seguridad, Falta de procedimientos, Falta de capacitación, Falta de indicadores y Falta de herramientas de control para el mantenimiento- que originan el $80 \%$ de problemas.

Se formulan planes de estandarización del proceso de servicio de mantenimiento, formatos e indicadores que permiten controlar y monitorear dichas actividades bajo el TPM. Logrando una reducción de S/350 000,00 anuales en el servicio de mantenimiento de molinos SAG.

Se concluye que el proyecto de mejora en el servicio de mantenimiento de molinos SAG es factible y rentable dado que presenta unos indicadores de evaluación, utilizando una tasa de descuento de $15 \%$; iguales a VAN de S/600 343,35 y una TIR de $430 \%$.

\section{REFERENCIAS}

[1] Acuña, B. (2019). Propuesta de mejora para la gestión de mantenimiento mediante la aplicación de herramientas del mantenimiento productivo total (TPM) en el área de tejeduría en una empresa textil. (Tesis de Licenciatura). Universidad Peruana de Ciencias Aplicadas, Lima, Perú. Recuperado https://repositorioacademico.upc.edu.pe/handle/10757/651599.

[2] Duffuaa, S., Raouf, A. \& Dixon, J. (2010). Sistemas de Mantenimiento. Planeamiento y Control. México: LIMUSA WILEY

[3] Ahuja, I. y Khamba, J. (2008). Total productive maintenance implementation in a manufacturing organization. Punjabi University, Patiala.

[4] Aguilar, R. (2018). Análisis y mejoras de la gestión del área de mantenimiento mecánico molienda procesos $\mathrm{C} 2$ de la planta concentradora de cobre de Sociedad Minera Cerro Verde Arequipa basado en la filosofía de mantenimiento productivo total. (Tesis de Licenciatura). Universidad Continental, Arequipa, Perú. Recuperado de: https://repositorio.continental.edu.pe/handle/20.500.12394/4970.

[5] Robbins, S. \& Judge, T. (2009). Comportamiento organizacional. (13 ${ }^{\mathrm{a}}$ ed.). México: Pearson Educación.

[6] Pinto, D. \& Mesa, J. (2011). Implementación de Plan Piloto de TPM en una Industria de Cerámica. (Tesis de Licenciatura). Universidad EAFIT, Medellín, Colombia. Recuperado de: https://repository.eafit.edu.co/bitstream/handle/10784/4362/DiegoPinto.

[7] Apaza, R. (2015). El modelo de mantenimiento productivo total TPM y su influencia en la productividad de la empresa minera Chama Perú E.I.R.L. (Tesis de Licenciatura). Universidad Andina, Juliaca, Perú. Recuperado de

http://repositorio.uancv.edu.pe/bitstream/handle/UANCV/438/TESIS.pdf 